\title{
Estudios empíricos del modelo sobre conocimiento didáctico- tecnológico del contenido (TPACK) en matemáticas, incluidos en bases bibliográficas internacionales
}

\author{
Susan B. Rivera-Robles ${ }^{1}$, Pedro A. Salcedo-Lagos ${ }^{2}$, Jorge R. Valdivia-Guzmán², y Ociel A. López-Jara ${ }^{3}$ \\ (1) Magister en Ciencias de la Educación, Facultad de Educación Universidad Católica de la Santísima Concepción, \\ Concepción, Chile (correo-e: sriverar@magisteredu.ucsc.cl). \\ (2) Departamento de Metodología de Investigación e Informática Educacional, Facultad de Educación Universidad de \\ Concepción, Concepción, Chile (correo-e: psalcedo@udec.cl; jvaldivi@udec.cl). \\ (3) Centro de Innovación y Desarrollo Docente, Dirección de Docencia, Universidad Católica de la Santísima \\ Concepción, Concepción, Chile (correo-e: olopez@ucsc.cl).
}

Recibido Ene. 22, 2021; Aceptado Mar. 19, 2021; Versión final Abr. 16, 2021, Publicado Ago. 2021

\section{Resumen}

El propósito de este estudio es realizar una revisión sistemática en base de datos de Web of Science (WOS), Scopus y Scielo sobre el enfoque del conocimiento didáctico-tecnológico del contenido (TPACK, en inglés) en docentes de matemática para estudios empíricos publicados desde el año 2018 hasta el 2020. Se caracteriza la investigación empírica desarrollada en el marco del modelo TPACK de docentes y futuros docentes de matemática y se describen los enfoques utilizados para identificar el TPACK del docente de matemática. La búsqueda se realizó en septiembre de 2020. Como criterio de inclusión se consideraron artículos con hallazgos empíricos sobre análisis cuantitativo y/o cualitativo del TPACK de profesores de matemática y artículos que utilicen el marco TPACK para el análisis de experiencias pedagógicas. Los hallazgos muestran variedad de enfoques para examinar el TPACK del docente. Se concluye que identificar el TPACK del maestro a través de evaluaciones del desempeño es más utilizado en las investigaciones que a través del autoinforme.

\section{Empirical studies of the model of technological pedagogical and content knowledge (TPACK) in mathematics, included in international bibliographic databases}

\begin{abstract}
The purpose of this study is to conduct a systematic review of the technological pedagogical and content knowledge (TPACK) approach of mathematics teachers for empirical studies published on the Web of Science (WOS), Scopus, and Scielo databases from the years 2018 until 2020. There are two defined objectives: 1) to characterize empirical research developed within the framework of the TPACK model for math teachers and 2) to describe the approaches used to identify the TPACK of math teacher. The databases searches were performed in September 2020. The following search criteria were used: 1) articles with empirical findings on quantitative and/or qualitative analysis of the TPACK of math teachers and 2) articles that use the TPACK framework for the analysis of pedagogical experiences. The results show a variety of approaches for examining the teacher's TPACK. In conclusion, identifying the teacher's TPACK through performance evaluations is more frequent in research than in self-reports.
\end{abstract}




\section{INTRODUCCIÓN}

La labor del docente es integrar el contexto, los saberes y el manejo de las Tecnologías de la Información y Comunicación (TIC), siendo la integración de las tecnologías indispensable en la educación actual (Fernández et al., 2016) debiendo implementarse de forma cotidiana (Sánchez-Otero et al., 2019). EI TPACK (Technological Pedagogical and Content Knowledge) es un modelo que permite integrar estos tres dominios. Está basado en el Conocimiento Didáctico del Contenido (PCK) de Shulman, quien afirma que el conocimiento de contenido es necesario para comprender y conocer el orden en cómo se pueden enseñar conceptos o temas, mientras que el conocimiento pedagógico permite conocer las diferentes formas en que se pueden enseñar los temas, tomando una mirada crítica en las ventajas y desventajas de cada enfoque, por lo que ambas son necesarias en el quehacer pedagógico (Gudmundsdottir y Shulman, 1987).

El modelo cuenta con un instrumento creado por Schmidt et al., (2009) que mide la autopercepción TPACK de los docentes mediante un cuestionario conformado por 58 ítems. El modelo TPACK complementa el conocimiento didáctico (PK), el conocimiento de contenido (CK) y el conocimiento tecnológico (TK) de los docentes (Koehler et al., 2015). Comúnmente se representa mediante un esquema con diagramas de Ven como se muestra en la Figura 1. Según Heitink et al., (2016) el modelo TPACK impacta de manera positiva en el área matemática, lo que podría desarrollar un proceso de integración TIC en esta especialidad. En los últimos años se han realizado una variedad de estudios empíricos donde se aplica el modelo TPACK para talleres que promueven la mejora en el área matemática (Salas-Rueda, 2018) o donde se aplica el cuestionario TPACK para medir la autopercepción de los docentes (Acikgul y Aslaner, 2019; Huang, 2018). Se han realizado algunas revisiones sistemáticas sobre estudios TPACK (Gür y Karamete, 2015; Voogt et al., 2012; Wilermark, 2017).

Stapf y Martin (2019) realizaron una revisión de literatura para investigaciones TPACK de docentes de matemática de primaria, por lo que no se han realizado revisiones sistemáticas donde se especifiquen las características de estudios basados en el modelo TPACK en el área de la matemática. Con lo expuesto anteriormente y con el fin de analizar estudios del modelo TPACK matemático en base de dato WOS, Scopus y Scielo, se realiza una revisión sistemática. Las preguntas de investigación que enmarcarán esta revisión son: ¿Cuáles son las características generales de los artículos recientes de TPACK en el área de la matemática en las bases de datos WOS, Scopus y Scielo? y ¿Qué enfoques se utilizan para identificar el TPACK del docente de matemática? Para responder a estas preguntas se establecen los siguientes objetivos: 1) Caracterizar la investigación empírica desarrollada en el marco del modelo TPACK de docentes y futuros docentes de matemática y 2) Describir los enfoques utilizados para identificar el TPACK del docente de matemática.

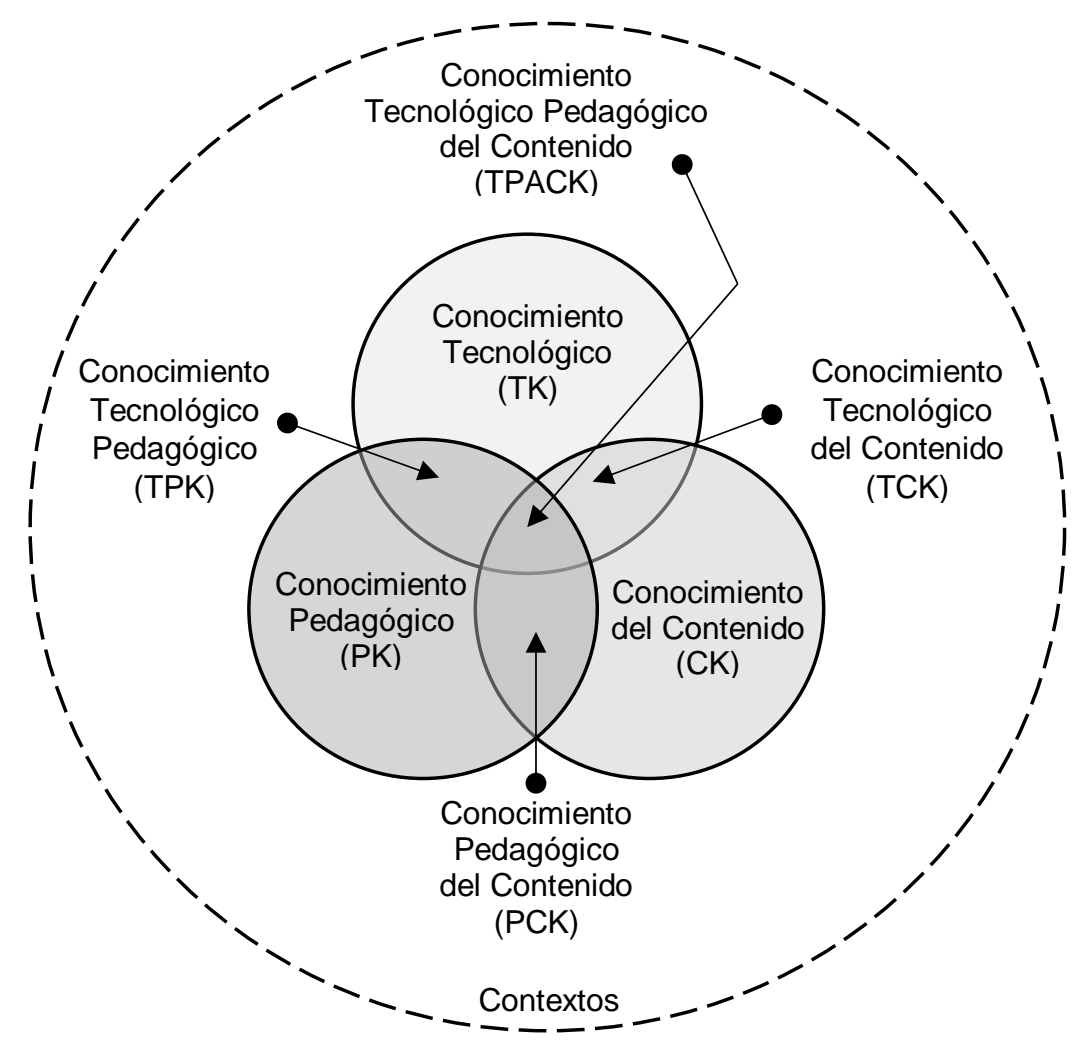

Fig. 1: Dominios del modelo TPACK (Adaptada de Koehler, 2015) 


\section{MÉTODO}

En el siguiente apartado se aborda el desarrollo de la revisión sistemática desde la búsqueda de los artículos, los criterios de inclusión y la selección final de los artículos a analizar.

\section{Selección de los artículos y búsqueda de artículos}

Para restringir la búsqueda, se seleccionaron tres bases de datos donde se considera que existen revistas de alto impacto: WOS Scopus y Scielo. Las palabras clave utilizadas para la búsqueda fueron ((TPACK OR TPCK) AND (Mathematic* OR Mathematical OR Math)) considerando artículos publicados entre 2018 a 2020. Dicha búsqueda se realizó en septiembre de 2020 y arrojo 108 artículos en la base de datos WOS, 23 artículos en la base de datos Scopus y 6 artículos en la base de datos Scielo, dando un total de 137 artículos. Para la selección los artículos se tomaron en cuenta los criterios de inclusión: a) El artículo debe contener hallazgos empíricos sobre análisis cuantitativo y/o cualitativo del TPACK de profesores de matemática; b) El artículo utiliza el marco TPACK para el análisis de experiencias pedagógicas de profesores de matemática. Los títulos y resúmenes de los 137 artículos fueron examinados manual y sistemáticamente por los autores. En caso de que hubiera dudas, el artículo se guardó para una lectura completa. Esto condujo a la lectura de 28 artículos por parte de los autores utilizando los mismos criterios de inclusión. Finalmente se seleccionaron 32 artículos para un análisis en profundidad. En la Figura 2 se muestra el flujograma de búsqueda y selección de las fuentes analizadas desde la base de datos WOS, Scopus y Scielo.

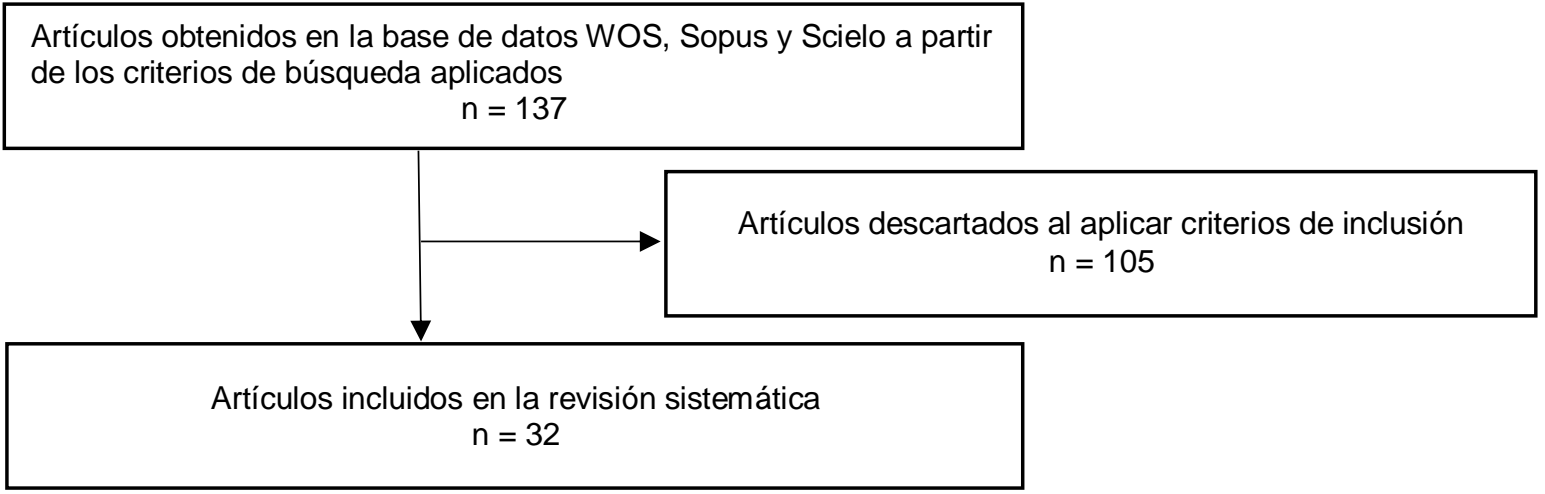

Fig. 2: Flujograma de búsqueda y selección de las fuentes analizadas desde la base de datos WOS, Scopus y Scielo

Entre los 105 artículos descartados, en su mayoría utilizaba el modelo TPACK como base teórica para otro tipo de investigaciones o bien para recomendar el uso del modelo en futuras investigaciones. También se encontraron revisiones sistemáticas de estudios TPACK en otras bases de datos y en años anteriores a los incluidos en este artículo.

\section{Parámetros de análisis considerados}

En la Tabla 1 se muestra un análisis de contenido el cual incluyo un análisis cuantitativo y cualitativo de los 32 artículos. Se realizó un análisis desde diversas perspectivas, por lo que se establecieron ciertos parámetros de análisis con el fin de categorizar y analizar las características generales de los artículos.

Tabla 1: Descripción de Parámetros de Análisis

\begin{tabular}{|l|l|}
\hline \multicolumn{1}{|c|}{ Parámetro de análisis } & \multicolumn{1}{c|}{ Descripción } \\
\hline Fuente & Es la referencia al artículo que contiene el estudio \\
\hline Index & Tipo de indexación de la revista donde se publicó el artí́culo \\
\hline País & País donde se realiza el estudio \\
\hline Año & Año de publicación del artículo \\
\hline Método & Describe el tipo de método utilizado: Cuantitativa, cualitativa o mixta. \\
\hline Tamaño Muestra & Cantidad de participantes del estudio \\
\hline Objeto de estudio & Docentes involucrados en el estudio (futuros docentes, docentes en ejercicio o mixto) \\
\hline Identificación TPACK & $\begin{array}{l}\text { Describe si la investigación examina el TPACK como conocimiento, como } \\
\text { competencia, o como marco para guiar otro tipo de investigaciones. }\end{array}$ \\
\hline
\end{tabular}


Posteriormente, los artículos se analizaron según la identificación de Willermark (2017), el cual categoriza si las investigaciones capturan el TPACK con un autoinforme (cuestionario) o evaluando el desempeño del quehacer pedagógico, esto con el objetivo de identificar si los artículos estudian el TPACK como conocimiento o competencia. Tanto el autoinforme como el desempeño de actividades docente se subdivide en tres categorías, que según Willermark (2017) serían las siguientes:

Autoinforme. Se subdivide en: (a) Conocimiento TPACK general: Situaciones donde los docentes estiman su percepción TPACK, tanto con el uso del cuestionario TPACK como con declaraciones tales como "me considero bueno en el uso tecnológico"; (b) Conocimiento TPACK específico: Situaciones donde se sitúa y evalúa un TPACK específico (Como TPACK-G, T-TPACK, TPACK-S, etc.) y en situaciones reales o ficticias específicas; (c) Conocimiento TPACK experimentado: Situaciones donde los docentes informan su percepción TPACK luego de experiencias diversas como talleres, actividades de enseñanza, cursos o intervenciones.

Desempeño de actividad docente. Circunstancias donde el desempeño es percibido por un agente externo mediante análisis de contenidos, entrevistas u observación. Se subdivide en: (a) Planificación de actividad docente: Se estudia el TPACK docente en el diseño y planificación de actividades pedagógicas; (b) Implementación de actividad docente: Se estudia el TPACK docente durante o después de implementar una actividad pedagógica; (c) Evaluación de actividad docente: Se evalúa el TPACK docente durante la reflexión crítica luego de una actividad pedagógica.

Para Willermark (2017), el autoinforme del conocimiento TPACK general indica que se estudió el TPACK solamente como conocimiento, el estudio del TPACK específico mueve un poco la investigación hacia el TPACK como competencia, así como el estudio del TPACK experimentado mueve aún más la investigación hacia ese ámbito. Estudiar el desempeño de un profesor en su planificación e implementación se acercan aún más a la competencia TPACK, siendo el estudio de la evaluación de la actividad docente exclusivamente una investigación de TPACK como competencia, tal como se muestra en la Figura 3. Adicionalmente se agregó la categoría "Guía" la cual indica que el TPACK se utilizó como modelo o apoyo para otras investigaciones empíricas clasificando otros instrumentos o experiencias según los dominios del TPACK.

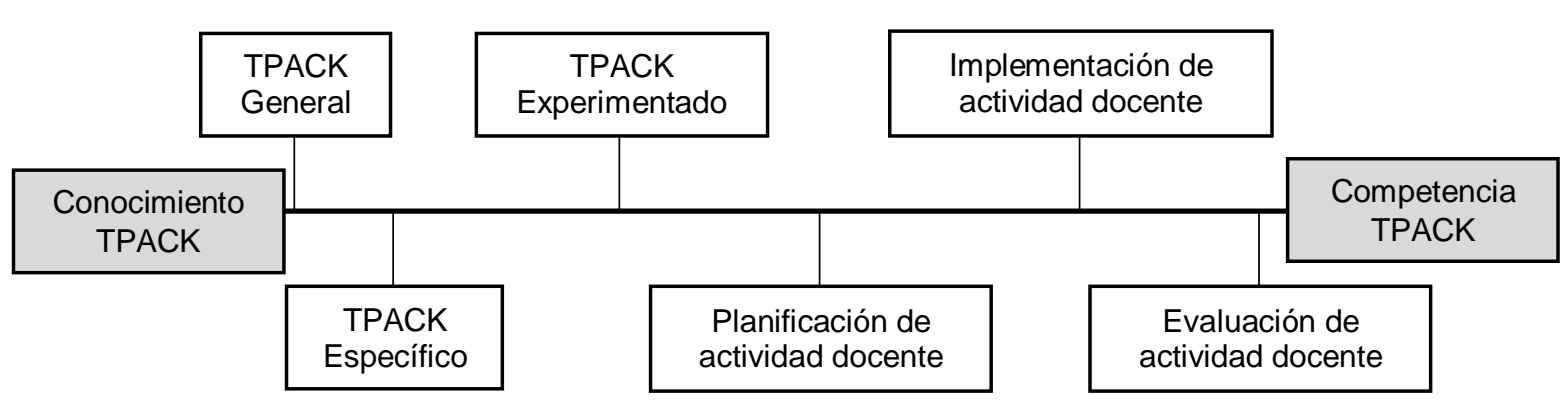

Fig. 3: Categorización sobre perspectivas TPACK (Adaptada de Willermark, 2017).

\section{RESULTADOS}

En el siguiente apartado se muestran los hallazgos obtenidos a partir de los objetivos y parámetros establecidos, presentando en primer lugar las características generales de las investigaciones para luego identificar el método utilizado en los artículos. En la Tabla 2 se muestra una descripción general de los artículos revisados.

\section{Características generales}

Según los resultados exhibidos en la Tabla 2, el 40,6\% de las investigaciones utilizaron metodologías cuantitativas como estudios correlacionales (Özgen y Narlı, 2020; Başaran, 2020), el 53,1\% de las investigaciones utilizaron metodologías cualitativas, donde el estudio de caso y análisis de contenido fueron las técnicas más utilizadas (Za'ba et al., 2020; Koh, 2019), y el 6,3\% de las investigaciones utilizaron una metodología mixta (De Freitas y Spangenberg, 2019; Young et al., 2019). Los estudios cualitativos utilizaron aproximadamente 10 docentes para las investigaciones, mientras que en los estudios cuantitativos se utilizaron aproximadamente 133 profesores y en estudios mixtos aproximadamente 47 docentes de muestra. 
Tabla 2: Características generales de estudios TPACK de profesores de matemática

\begin{tabular}{|c|c|c|c|c|c|c|c|c|c|}
\hline \multirow[t]{2}{*}{$N$} & \multirow[t]{2}{*}{ Fuente } & \multirow[t]{2}{*}{ País } & \multicolumn{3}{|c|}{ Método } & \multicolumn{3}{|c|}{ Objeto de estudio } & \multirow[t]{2}{*}{ Nivel } \\
\hline & & & Cuantitativo & Cualitativo & Mixto & $\begin{array}{l}\text { Futuros } \\
\text { Profesores }\end{array}$ & $\begin{array}{c}\text { Profesores en } \\
\text { ejercicio }\end{array}$ & Mixto & \\
\hline 1 & \begin{tabular}{|l|} 
Kaplon-Schilis y \\
Lyublinskaya, 2020
\end{tabular} & EEUU & $x$ & & & $x$ & & & Primaria \\
\hline 2 & Za'ba et al., 2020 & Malasia & & $x$ & & $x$ & & & Media \\
\hline 3 & Özgen y Narlı, 2020 & Turquía & $x$ & & & $x$ & & & Media \\
\hline 4 & Başaran, 2020 & Turquía & $x$ & & & $x$ & & & Secundaria \\
\hline 5 & Cenich, 2020 & Argentina & & $x$ & & & $X$ & & Secundaria \\
\hline 6 & $\begin{array}{l}\text { Gutiérrez y } \\
\text { Henríquez, } 2020\end{array}$ & Portugal & & $x$ & & $x$ & & & Media \\
\hline 7 & $\begin{array}{l}\text { Khoza y Biyela, } \\
2020\end{array}$ & Sudáfrica & & $x$ & & $x$ & & & Media \\
\hline 8 & $\begin{array}{l}\text { Backfisch et al., } \\
2020\end{array}$ & Alemania & $x$ & & & & & $x$ & Media \\
\hline 9 & $\begin{array}{l}\text { Salas-Rueda et al., } \\
2020\end{array}$ & México & $\mathrm{x}$ & & & $x$ & & & Media \\
\hline 10 & $\begin{array}{l}\text { Zambak y Tyminski, } \\
2020\end{array}$ & EEUU & & $x$ & & $x$ & & & Media \\
\hline 11 & \begin{tabular}{|l|} 
De Freitas y \\
Spangenberg, 2019
\end{tabular} & Sudáfrica & & & $x$ & $x$ & & & Media \\
\hline 12 & Koh, 2019 & Singapur & & $x$ & & & $\mathrm{X}$ & & Primaria \\
\hline 13 & $\begin{array}{l}\text { Tsouccas y } \\
\text { Meletiou- } \\
\text { Mavrotheris, } 2019\end{array}$ & Chipre & & $x$ & & & $x$ & & Primaria \\
\hline 14 & Young et al., 2019 & EEUU & & & $x$ & & $X$ & & Secundaria \\
\hline 15 & Salas-Rueda, 2019 & México & $\mathrm{X}$ & & & $x$ & & & Secundaria \\
\hline 16 & $\begin{array}{l}\text { Acikgul y Aslaner, } \\
2019 a\end{array}$ & Turquía & $x$ & & & $x$ & & & Primaria \\
\hline 17 & $\begin{array}{l}\text { Acikgul y Aslaner, } \\
2019 b\end{array}$ & Turquía & $x$ & & & $x$ & & & Primaria \\
\hline 18 & Harits, 2019 & Surakarta & & $\mathrm{X}$ & & & $\mathrm{X}$ & & Secundaria \\
\hline 19 & $\begin{array}{l}\text { Yildiz y Gokcek, } \\
2018\end{array}$ & Turquía & & $x$ & & $x$ & & & Secundaria \\
\hline 20 & $\begin{array}{l}\text { Loong y Herbert, } \\
2018\end{array}$ & Australia & & $x$ & & $x$ & & & Primaria \\
\hline 21 & Huang, 2018 & China & $x$ & & & & $x$ & & Universidad \\
\hline 22 & Akyuz, 2018 & Turquía & $x$ & & & $x$ & & & Media \\
\hline 23 & Honey, 2018 & Inglaterra & & $x$ & & & & $x$ & Primaria \\
\hline 24 & \begin{tabular}{|l} 
Caniglia y \\
Meadows, 2018
\end{tabular} & EEUU & & $x$ & & & $x$ & & Secundaria \\
\hline 25 & Saralar et al., 2018 & Turquía & & $x$ & & & $x$ & & Media \\
\hline 26 & $\begin{array}{l}\text { Watson y } \\
\text { Enderson, } 2018\end{array}$ & EEUU & & $X$ & & $\mathrm{X}$ & & & Secundaria \\
\hline 27 & $\begin{array}{l}\text { Meletiou- } \\
\text { Mavrotheris et al., } \\
2018\end{array}$ & Chipre & & $x$ & & & $\mathrm{X}$ & & Primaria \\
\hline 28 & Lai y Lin, 2018 & Taiwán & $x$ & & & & $\mathrm{X}$ & & Secundaria \\
\hline 29 & Yan et al., 2018 & China & & $x$ & & & & $x$ & Secundaria \\
\hline 30 & $\begin{array}{l}\text { Alizadeh-Jamal et } \\
\text { al., } 2018\end{array}$ & Irán & $x$ & & & & $x$ & & Secundaria \\
\hline 31 & He et al., 2018 & China & $\mathrm{X}$ & & & & $\mathrm{X}$ & & Media \\
\hline 32 & Muhtadi et al., 2018 & Indonesia & & $\mathrm{X}$ & & & $x$ & & Media \\
\hline \multicolumn{3}{|c|}{ Total, de artículos } & 13 & 17 & 2 & 16 & 13 & 3 & \\
\hline
\end{tabular}


El $50 \%$ de los estudios utilizaron como objeto de estudio a futuros profesores, donde el, $50 \%$ pertenecen a estudios cualitativos, $43,8 \%$ a estudios cuantitativos y $6,2 \%$ a estudios mixtos. El $40,6 \%$ de los estudios tenían como objeto de estudio a docentes en ejercicio, donde el $61,5 \%$ pertenece a estudios cualitativos; $30,8 \%$ a estudios cuantitativos y $7,7 \%$ a estudios mixtos. El $9,4 \%$ de los estudios tuvo como objeto de estudio tanto docentes en ejercicio como futuros docentes, donde el $66,7 \%$ pertenece a estudios cualitativos y el $33,3 \%$ a estudios cuantitativos. Por su parte, un $25 \%$ de los artículos consideró a docentes o futuros docentes de educación primaria, un $34 \%$ a profesores o futuros profesores de educación secundaria, un $38 \%$ a docentes de niveles medios y un $3 \%$ a profesores universitarios.

Las investigaciones cualitativas principalmente buscaban caracterizar $y / 0$ analizar los componentes del modelo mediante observación de clases, revisión de sus planificaciones o análisis del discurso docente mediante redacción de tareas matemática o entrevistas. Las investigaciones cuantitativas principalmente analizaban los componentes del modelo TPACK y sus variantes (TPACK -21, TTPACK, MTPACK) para correlacionarlas entre ellas y determinar predictores, buscando desarrollar, en algunos casos, nuevos instrumentos para describir los componentes TPACK. También hay investigaciones que aplican pre y post donde se capacita a los docentes en el uso de una herramienta tecnológica (Geogebra). Las investigaciones mixtas son de intervención, donde se aplica el cuestionario TPACK, pero también se analizan tareas matemáticas, clases y planificaciones para relacionar su autopercepción con la eficacia al integrar tecnologías en el aula

\section{Características específicas}

La tabla 3 agrupa los 32 artículos de la revisión según sus características investigativas donde también se indica el aporte en el área educativa y en nuevas investigaciones o publicaciones futuras.

Tabla 3: Características específicas de estudios TPACK de profesores de matemática

\begin{tabular}{|c|c|c|}
\hline Artículos & Característica & Aportes en el ámbito educativo matemático \\
\hline $\begin{array}{l}\text { Kaplon-Schilis y } \\
\text { Lyublinskaya, 2020; Özgen } \\
\text { y Narlı, 2020; Başaran, } \\
\text { 2020; Akyuz, 2018; He et } \\
\text { al., 2018 }\end{array}$ & $\begin{array}{l}\text { Investigan los constructos del } \\
\text { TPACK y los analizan para ver si } \\
\text { existe correlación y/o dependencia, } \\
\text { desarrollando, en algunos casos, } \\
\text { nuevos instrumentos para describir } \\
\text { los componentes del TPACK }\end{array}$ & $\begin{array}{l}\text { Puede existir o no correlación entre los } \\
\text { constructos TPACK, todo dependerá del contexto } \\
\text { donde se realice la investigación, por lo que es } \\
\text { importante tener en cuenta que siempre se debe } \\
\text { hacer un análisis de correlación si se desea } \\
\text { analizar en profundidad el TPACK de los } \\
\text { docentes. Por otro lado, dentro del marco TPACK } \\
\text { se distinguen cuatro dominios adicionales (Core, } \\
\text { Tech, Tpack-P y Tpack-C) }\end{array}$ \\
\hline $\begin{array}{l}\text { Za'ba et al., 2020; Khoza y } \\
\text { Biyela, 2020; de Freitas y } \\
\text { Spangenberg, 2019; Young } \\
\text { et al., 2019; Acikgul y } \\
\text { Aslaner, 2019a; Yildiz y } \\
\text { Gokcek, 2018; Alizadeh- } \\
\text { Jamal et al., 2018 }\end{array}$ & $\begin{array}{l}\text { Se analiza el TPACK de docentes } \\
\text { antes y después de alguna } \\
\text { intervención (Talleres, capacitación, } \\
\text { etc.) donde se les enseño alguna } \\
\text { herramienta tecnológica }\end{array}$ & $\begin{array}{l}\text { En la mayoría de las intervenciones el TPACK } \\
\text { docente se vio beneficiado, sin embargo, los } \\
\text { docentes siguieron presentando mayor } \\
\text { conocimiento pedagógico y de contenido que } \\
\text { conocimiento tecnológico }\end{array}$ \\
\hline $\begin{array}{l}\text { Cenich, 2020; Gutierrez y } \\
\text { Herniquez, 2020; Backfisch } \\
\text { et al., 2020; Honey, 2018; } \\
\text { Saralar et al., 2018; Watson } \\
\text { y Enderson, 2018; Muhtadi } \\
\text { et al., } 2018\end{array}$ & $\begin{array}{l}\text { EI TPACK es utilizado como guía } \\
\text { para evaluar el desempeño en } \\
\text { clases de los docentes o bien tareas } \\
\text { matemáticas planificadas. También } \\
\text { se busca caracterizar el TPACK } \\
\text { docente mediante la observación de } \\
\text { clase o el modelado de una } \\
\text { situación pedagógica. }\end{array}$ & $\begin{array}{l}\text { La competencia TPACK podría considerarse } \\
\text { como atributos fundamentales para los futuros } \\
\text { profesores de matemáticas, donde la } \\
\text { consolidación y desarrollo TPACK es una } \\
\text { articulación de múltiples dominios, conocimientos } \\
\text { complejos, dinámicos y flexibles. Sin embargo, } \\
\text { los docentes de matemática se consideran, } \\
\text { habitualmente, con bajo conocimiento TPACK, } \\
\text { donde los docentes con menor experiencia o de } \\
\text { niveles bajo, suelen tener menor manejo } \\
\text { tecnológico. Se descubre que a medida que los } \\
\text { docentes utilizan tecnología en sus clases, el } \\
\text { dominio sobre estas va aumentando, lo que } \\
\text { implica que el bajo maneto tecnológico se debe } \\
\text { también al poco uso de este. }\end{array}$ \\
\hline
\end{tabular}


Tabla 3: continuación.

\begin{tabular}{|c|c|c|}
\hline Artículos & Característica & Aportes en el ámbito educativo matemático \\
\hline $\begin{array}{l}\text { Salas-Rueda et al., 2020; } \\
\text { Tsouccas y Meletiou- } \\
\text { Mavrotheris, 2019; Salas- } \\
\text { Rueda, 2019; Meletiou- } \\
\text { Mavrotheris et al., 2018; } \\
\text { Yan et al., } 2018\end{array}$ & $\begin{array}{l}\text { Se utiliza el modelo TPACK } \\
\text { como guía para organizar, } \\
\text { diseñar o implementar } \\
\text { diversos programas de } \\
\text { mejora }\end{array}$ & $\begin{array}{l}\text { El modelo TPACK facilita el diseño, planificación y } \\
\text { organización de nuevas experiencias educativas por } \\
\text { medio de los conocimientos tecnológico, disciplinar y } \\
\text { pedagógico. Por consiguiente, este modelo es un } \\
\text { referente ideal para innovar el proceso de enseñanza y } \\
\text { aprendizaje, así como para estudiar y facilitar el } \\
\text { crecimiento profesional docente }\end{array}$ \\
\hline $\begin{array}{l}\text { Zambak y Tyminski, 2020; } \\
\text { Kho, 2019; Harits, 2019; } \\
\text { Loong y Herbert, 2018; } \\
\text { Huang, } 2018\end{array}$ & $\begin{array}{l}\text { Basándose en el modelo } \\
\text { TPACK buscan desarrollar o } \\
\text { comprobar la efectividad de } \\
\text { nuevas variantes del modelo } \\
\text { (TPACK-21, TPACK - T, } \\
\text { MTPACK) }\end{array}$ & $\begin{array}{l}\text { En primer lugar, se revela que existen cinco niveles de } \\
\text { conocimiento tecnológico matemático donde se puede } \\
\text { clasificar a los profesores de matemática. Por otro lado, el } \\
\text { TPMK (TPACK específico para docentes de matemática) } \\
\text { no capta las creencias de los profesores. En tercer lugar, } \\
\text { se confirma que el TPACK se ve afectado por la } \\
\text { experiencia del docente utilizando tecnología en el aula. } \\
\text { Finalmente, se devela que los docentes universitarios } \\
\text { carecen de conocimiento pedagógico y psicológico, lo cual } \\
\text { afecta negativamente en la formación docente. }\end{array}$ \\
\hline $\begin{array}{l}\text { Acikgul y Aslaner, } 2019 b ; \\
\text { Caniglia y Meadows, } \\
\text { 2018; Lai y Lin, } 2018\end{array}$ & $\begin{array}{l}\text { Se compara el TPACK } \\
\text { docente con otras variables } \\
\text { como la eficacia TPACK, } \\
\text { creencias centradas en el } \\
\text { estudiante } u \text { otros modelos. }\end{array}$ & $\begin{array}{l}\text { EI TPACK de los docentes no se relaciona con la eficacia } \\
\text { en el uso te tecnologías en aula, pero si con las creencias } \\
\text { centradas en el estudiante. }\end{array}$ \\
\hline
\end{tabular}

\section{Número de artículos publicados}

En la Figura 4 se muestra el flujo de cantidad de publicaciones desde 2018 de los 32 artículos analizados. Se observa que la mayor cantidad de artículos publicados fue en el año 2018, luego hay una disminución siendo el 2019 el año con menor cantidad de publicaciones y luego aumentando en 2020.

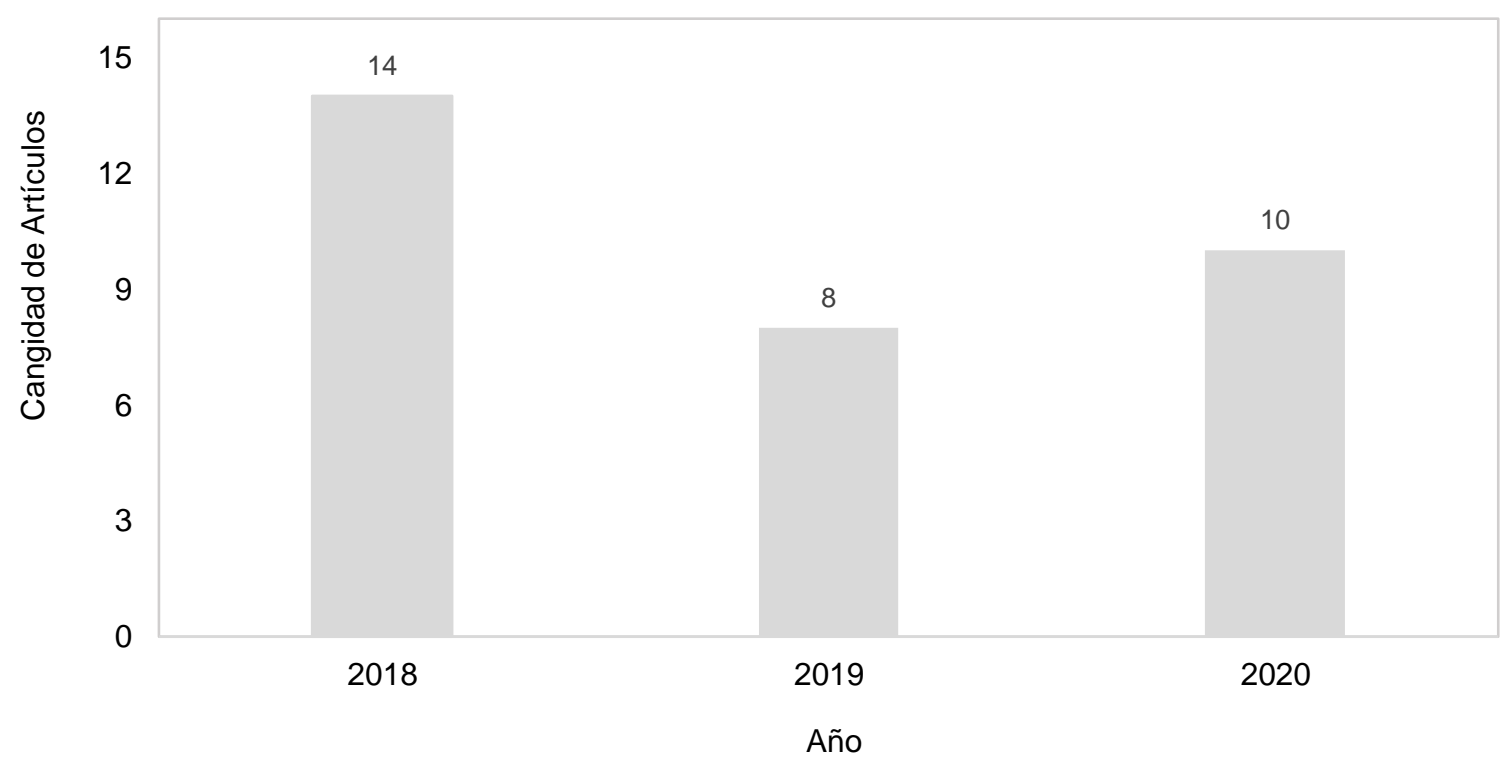

Fig. 4: Producción por año de estudios TPACK matemático en base de datos WOS, Scopus y Scielo 


\section{Bases de datos}

En la muestra seleccionada, la mayor cantidad de investigaciones sobre TPACK de docentes de matemática (75\%) se publicaron en revistas con indexación WOS, seguidas por revistas con indexación Scopus $(21,9 \%)$ teniendo la menor cantidad de artículos publicados con esta temática las revistas con indexación Scielo $(3,1 \%)$.

\section{Enfoques para la identificación TPACK}

Debido a que el TPACK del docente de matemática se determinó, en ocasiones, mediante varios instrumentos y técnicas, se decidió tomar en cuenta aquel que destacaba en el artículo y con el cual se daba preponderancia al análisis y conclusiones. En la Tabla 4 se muestra que con respecto al escenario - vía desempeño de actividad docente - se destacó la opción, "implementación de la actividad docente" (25\%). En lo referente al escenario - vía autoinforme - se observa que la elección "conocimiento TPACK general" es la que se destaca (25\%). No obstante, la minoría de los artículos utiliza el TPACK como un marco de apoyo o guía en el análisis de otros contextos

Tabla 4: Categorización de la identificación del docente TPACK

\begin{tabular}{|l|c|l|c|c|}
\hline \multicolumn{2}{|c|}{ Vía autoinforme } & \multicolumn{2}{c|}{ Vía desempeño de actividad docente } & Como Guía \\
\hline Conocimiento TPACK general & $25 \%$ & Planificación de la actividad docente & $18,8 \%$ & \\
\hline Conocimiento TPACK específico & $6,3 \%$ & Implementación de la actividad docente & $25 \%$ & \multirow{2}{*}{$6,2 \%$} \\
\hline Conocimiento TPACK experimentado & $15,6 \%$ & Evaluación de la actividad docente & $3,1 \%$ & \\
\hline Autoinforme total & $46,9 \%$ & Vía desempeño de actividad docente total & $46,9 \%$ & \\
\hline
\end{tabular}

Autoinforme. Conocimiento TPACK general. La técnica mayormente utilizada fue la aplicación del cuestionario TPACK original, traducido o adaptado. Con este cuestionario unas investigaciones correlacionaron o clasificaron los dominios TPACK entre si con el fin de profundizar en el constructo del modelo TPACK (Özgen y Narlı, 2020; Zambak y Tyminski, 2020). Otros artículos se centraron en identificar los conocimientos de contenido, pedagógicos y tecnológicos del docente de matemática (de Freitas y Spangenberg, 2019) o bien midieron las creencias y percepciones de los profesores (Lai y Lin, 2018). Sin embargo, no fue la única técnica utilizada, también se analizó el TPACK general mediante entrevistas o análisis de contenido con el fin de identificar creencias o conocimientos principalmente tecnológicos en los profesores de matemática (Watson y Enderson, 2018).

Autoinforme. Conocimiento TPACK específico. Las investigaciones buscan analizar un contexto específico donde se ponga en juego el TPACK del docente de matemática. Başaran (2020) estudia el TPACK-21, un modelo que busca identificar los componentes TPACK centrado en las habilidades del siglo XXI. En la investigación de Huang (2018) se explora la estructura del T-TPACK, en cual describe la relación entre el TPACK de los profesores de matemáticas y sus estudiantes en el proceso de enseñanza y aprendizaje, y en este caso, del pensamiento matemático.

Autoinforme. Conocimiento TPACK experimentado. En su mayoría, este tipo de investigaciones realizaron intervenciones para luego aplicar el cuestionario TPACK realizando un pre y post test (Acikgul y Aslaner, 2019) comparación de grupos (Backfisch et al., 2020) o contrastarlo con otros instrumentos utilizados luego de la experiencia (Young et al., 2019).

Planificación de la actividad docente. Por un lado, las investigaciones se dedicaron a analizar los documentos con los que planificaban los docentes para encontrar en ellos componentes de TPACK (Za'ba et al., 2020) o bien se analizaron grabaciones de entrevistas o textos de foros o chats (Gutiérrez y Henríquez, 2020). Por otro lado, se realizó un análisis de planeaciones o comentarios luego de la implementación de un taller o capacitación sobre alguna herramienta didáctica centrada en matemática (Tsouccas y Meletiou-Mavrotheris, 2019).

Implementación de la actividad docente. Tal como en el punto anterior, se analizó la manera en cómo el docente realiza el proceso de enseñanza y aprendizaje ya sea en su entorno común o bien luego de un taller de especialización, unas investigaciones observaron clases reales o bien realizaron entrevistas inmediatamente luego de estas (Yildiz y Gokcek, 2018) y otras pusieron a los docentes en un entorno ficticio para implementar una clase (Khoza y Biyela, 2020; Kho, 2019).

Evaluación de la actividad docente. Aunque son escasas, estas investigaciones realizan reflexiones junto con los objetos de estudio para evaluar el desempeño luego de la implementación. Meletiou-Mavrotheris et al., (2018) investiga, por ejemplo, la incorporación de juegos en la estadística temprana donde la reflexión impactó 
de manera positiva, pues los docentes involucrados transfirieron la competencia TPACK en su práctica habitual.

Guía. Unas investigaciones utilizaron el TPACK, o adaptación de este, para guiar el marco teórico o para organizar otros instrumentos de recolección de datos (Salas-Rueda et al., 2020). Así mismo, se analizaron las experiencias docentes de aula bajo otros parámetros para luego realizar recomendaciones en torno al modelo TPACK (Salas-Rueda, 2019).

\section{DISCUSIÓN}

En cuanto al primer objetivo sobre caracterizar la investigación empírica desarrollada en el marco del modelo TPACK de docentes y futuros docentes de matemática, sin dudas la cantidad de artículos referentes al TPACK ha aumentado a través del tiempo, donde cada investigación tiene características propias de su contexto, diferenciándose por este motivo o bien por la manera en cómo se concibe el TPACK. En primera instancia es recomendable discutir la relación entre los resultados de otras revisiones sistemáticas con las de esta investigación, tal como se realiza en revisiones sistemáticas de otros ámbitos (Zambrano et al, 2019). Gür y Kamarete (2015) analizan los temas de los artículos sobre TIC y TPACK, sin embargo, no analizan si los artículos conciben el TPACK como competencia o conocimiento. Por su lado, Voogt et al., (2012) investigaron la base teórica y el uso práctico de TPACK en los artículos revisados encontrando diversas concepciones sobre el TPACK, donde apenas se encontraron nociones acerca de TPACK en los dominios temáticos.

La revisión de Stapf y Martin (2019) analiza tanto el desarrollo TPACK como el TPACK matemático de docentes previos al servicio a quienes se les realizaron intervenciones sobre didáctica matemática, dando como conclusión la importancia de comprender los constructos del modelo TPACK en el desarrollo docente, sin embargo, no analiza a profundidad las actividades realizadas en las investigaciones revisadas. Willermark (2017) es quien analiza las caracterizaciones del TPACK en su revisión sistemática, sus resultados afirman que existen más investigaciones realizadas vía autoinforme que sobre el desempeño de actividad docente. Estos resultados no coinciden con los hallados en esta revisión, pues los artículos sobre TPACK en docentes de matemática se centrarán en analizar más el desempeño de actividades que el autoinforme. Esto podría deberse a que las investigaciones centradas en docentes de matemática buscan analizar más la competencia TPACK que el conocimiento TPACK.

Respecto al segundo objetivo sobre describir los enfoques utilizados para identificar el TPACK del docente de matemática, se identificaron dos: (1) identificación TPACK vía autoinforme; o (2) identificación TPACK vía desempeño de actividad docente. No se incluye la utilización del marco TPACK como guía o apoyo para otros instrumentos $\mathrm{y} / \mathrm{o}$ experiencias pues en estas investigaciones no se identificó específicamente el TPACK docente.

(1) Identificación TPACK vía autoinforme. Las investigaciones que optaron por este camino tenían, en su mayoría, muestras grandes $(>30)$, por lo que permitir que el mismo docente pudiese evaluar su manejo pedagógico, tecnológico y de contenido, ya sea en un solo momento o bien luego de una intervención, es un modo eficiente de recopilar datos, sin embargo, varios estudios confirman que la autopercepción TPACK de los docentes de matemática no tiene relación necesaria con la eficacia en el uso de herramientas tecnológicas para la enseñanza (Acikgul y Aslaner, 2019; Huang, 2018).

(2) Identificación TPACK vía desempeño de actividad docente. Aquellas investigaciones que decidieron seguir este camino son en su mayoría cualitativas, pues identificar la competencia en un gran número de docentes no es una tarea sencilla, sobre todo cuando se requiere evaluar el desempeño en escenarios reales. Pese a que los resultados son difícilmente generalizables, pueden ser de gran ayuda en las comunidades escolares o universitaria sobre todo cuando se logra realizar una reflexión profunda sobre el TPACK de los participantes (Meletiou-Mavrotheris et al., 2018).

Por tanto, la revisión sistemática a los estudios de TPACK en matemática en la base de datos WOS, Scopus y Scielo muestra que la investigación está avanzando hacia concepciones más profundas sobre el TPACK, debido a que, de a poco, se está comenzando a medir el TPACK como competencia por sobre el TPACK como conocimiento, lo que conlleva a mayor reflexión y autocritica por parte de los docentes. La investigación presenta algunas limitaciones como lo es las bases de datos consultadas, pues pese a que se consideran de alto impacto, se dejaron de lado artículos con otro tipo de indexación. Por lo expuesto anteriormente, como recomendación a futuras investigaciones sugerimos: (1) Enfocar la investigación en la evaluación del desempeño docente, es decir, en la reflexión y autocritica en momentos clave de la labor pedagógica; (2) utilizar ambos tipos de enfoques en las investigaciones, donde se mida tanto el conocimiento TPACK como la competencia, esto con el fin de realizar una investigación completa; (3) esclarecer una conceptualización TPACK clara en las investigaciones, pues este puede ir variando de acuerdo al contexto siendo necesario profundizar en las definiciones (Voogt et al., 2012). 


\section{CONCLUSIONES}

Según los resultados y la discusión presentada en la investigación además de la comparación de investigaciones similares, se puede concluir que: 1) la mayoría de las investigaciones sobre TPACK en docentes de matemática se han publicado en revistas con indexación WOS, manteniendo su cantidad de publicaciones a través del tiempo; 2) los enfoques en los que se enmarcan las investigaciones TPACK para docentes de matemática esta aún dividida entre conocimiento y competencia; y 3) escasean los artículos donde se promueva la reflexión por parte de los sujetos de estudio luego de alguna experiencia pedagógica.

\section{AGRADECIMIENTOS}

Esta investigación se desarrolla en el marco del proyecto FONDECYT 1201572 (2020-2024): "Evaluación de la integración pedagógica de las tecnologías de la información y comunicación en el aula de matemática y de lenguaje y comunicación, desde el modelo TPACK basado en competencias".

\section{REFERENCIAS}

Açikgul, K., y Aslaner, R., Investigation relations between the technological pedagogical content knowledge efficacy levels and self-efficacy perception levels of pre-service mathematics teachers, Çukurova University Faculty of Education Journal, 48(1), 1-31 (2019)

Backfisch, I., Lachner, A., y otros tres autores, Professional knowledge or motivation? investigating the role of teachers' expertise on the quality of technology-enhanced lesson plans, https://doi.org/10.1016/j.learninstruc.2019.101300, Learning and Instruction, 66, 101300 (2020)

Başaran, B., Investigating science and mathematics teacher candidate's perceptions of TPACK-21 based on 21st century skills, https://doi.org/10.17051/ilkonline.2020.763851, Elementary Education Online, 19(4), 2212-2226 (2020)

De Freitas, G., y Spangenberg, E. D., Mathematics teachers' levels of technological pedagogical content knowledge and information and communication technology integration barriers, https://doi.org/10.4102/pythagoras.v40i1.431, Pythagoras, 40(1), 1-13 (2019)

Fernández, J. C., Fernández, M. C., y Cebreiro, B., Desarrollo de un cuestionario de competencias en TIC para profesores de distintos niveles educativos, https://doi.org/10.12795/pixelbit.2016.i48.09, Pixel-Bit: Revista de Medios y Educación, 48, 135-148 (2016)

Gudmundsdottir, S., y Shulman, L., Pedagogical content knowledge in social studies, https://doi.org/10.1080/0031383870310201, Scandinavian Journal of Educational Research, 31(2), 59-70 (1987)

Gür, H., y Kamarete, A., A short review of TPACK for teacher education, https://doi.org/10.5897/ERR2014.1982, Educational Research and Reviews, 10(7), 777-789 (2015)

Gutiérrez-Fallas, L., y Henriques, A., O TPACK de futuros professores de matemática numa experiência de formação, https://doi.org/10.12802/relime.20.2322, Revista Latinoamericana de Investigacion en Matematica Educativa-Relime, 23(2), 175-202 (2020)

Heitink, M., Voogt, J., y otros tres autores, Teachers' professional reasoning about their pedagogical use of technology, https://doi.org/10.1016/j.compedu.2016.05.009, Computers \& Education, 101, 70-83 (2016)

Huang, Z., theoretical analysis of TPACK knowledge structure of mathematics teachers based on T-TPACK mode, https://doi.org/10.12738/estp.2018.5.103, Educational Sciences: Theory \& Practice, 18(5), 2044-2053 (2018)

Khoza, S. B., y Biyela, A. T., Decolonising technological pedagogical content knowledge of first year mathematics students, https://doi.org/10.1007/s10639-019-10084-4, Education and Information Technologies, 25, 2665-2679 (2019)

Koehler, M. J., Mishra, P. y Cain, W., ¿Qué son los saberes tecnológicos y pedagógicos del contenido (TPACK)?, Virtualidad, Educación y Ciencia, 6(10), 9-23, (2015)

Koh, J. H. L., Articulating teachers' creation of technological pedagogical mathematical knowledge (TPMK) for supporting mathematical inquiry with authentic problems, https://doi.org/10.1007/s10763-018-9914-y, International Journal of Science and Mathematics Education, 17(6), 1195-1212 (2019)

Lai, T. L., y Lin, H. F., An investigation of the relationship of beliefs, values and technological pedagogical content knowledge among teachers, https://doi.org/10.1080/1475939X.2018.1496137, Technology, Pedagogy and Education, 27(4), 445-458 (2018)

Meletiou-Mavrotheris, M., Paparistodemou, E., y Tsouccas, L., Integrating games into the early statistics classroom: teachers' professional development on game-enhanced learning, https://doi.org/10.1007/978-981-13-1044-7_16, In Statistics in Early Childhood and Primary Education, 1, Springer Nature Singapore Pte Ltd., 275-293, Singapur (2018)

Özgen, K., y Narli, S., Intelligent data analysis of interactions and relationships among technological pedagogical content knowledge constructs via rough set analysis, https://doi.org/10.30935/cet.646769, Contemporary Educational

Technology, 11(1), 77-98 (2020) 
Salas-Rueda, R. A., Gamboa-Rodríguez, F., Salas-Rueda, É. P., y Salas-Rueda, R. D., Diseño de una aplicación web para el proceso educativo sobre el uso del logaritmo en el campo de las matemáticas financieras, https://doi.org/10.17851/1983-3652.13.1.65-81, Texto Livre: Linguagem e Tecnologia, 13(1), 65-81 (2020)

Sánchez-Otero, M., García-Guiliany, J., Steffens-Sanabria, E., y Palma, H. H., Estrategias pedagógicas en procesos de enseñanza y aprendizaje en la educación superior incluyendo tecnologías de la información y las comunicaciones, http://dx.doi.org/10.4067/S0718-07642019000300277, Información Tecnológica., 30(3), 277-286 (2019)

Salas-Rueda, R. A., Construction and evaluation of a web application for the educational process on normal distribution considering the science of data and machine learning, https://doi.org/10.25304/rlt.v27.2085, Research in Learning Technology, 27 (2019)

Schmidt, D., Baran, E., y otros cuatro autores, Technological pedagogical content knowledge (TPACK): The development and validation of an assessment instrument for preservice teachers,

https://doi.org/10.1080/15391523.2009.10782544, Journal of Research on Technology in Education, 42(2), $123-149$. (2009)

Stapf, K., y Martin, B., TPACK + Mathematics: A review of current TPACK literature, International Journal on Integrating Technology in Education, 8(3), 13-20. (2019)

Tsouccas, L. F., y Meletiou-Mavrotheris, M., Enhancing In-service primary teachers' technological, pedagogical and content knowledge on mobile mathematics learning, http://dx.doi.org/10.4018/IJMBL.2019070101, International Journal of Mobile and Blended Learning (IJMBL), 11(3), 1-18 (2019)

Voogt, J., Fisser, P., Pareja, N., y Tondeur, J., Technological pedagogical content knowledge - a review of the literature, https://doi.org/10.1111/j.1365-2729.2012.00487.x, Journal of Computer Assisted Learning, 29(2), 109-121 (2012)

Watson, G. S., y Enderson, M. C., Preparing teachers to use excelets: developing creative modeling experiences for secondary mathematics students, https://doi.org/10.1007/978-3-319-72381-5_8, In Creativity and Technology in Mathematics Education, 1, Springer, 203-231 (2018)

Willermark, S., Technological Pedagogical and Content Knowledge: A Review of Empirical Studies Published From 2011 to 2016, https://doi.org/10.1177/0735633117713114, Journal of Educational Computing Research, 56(3), 315-343 (2017)

Yildiz, H., y Gokcek, T., The development process of a mathematic teacher's technological pedagogical content knowledge, European Journal of Educational Research, 7(1), 9-29 (2018)

Young, J. R., Young, J., Hamilton, C., y Pratt, S. S., Evaluating the effects of professional development on urban mathematics teachers TPACK using confidence intervals, http://dx.doi.org/10.17583/redimat.2019.3065, REDIMAT, 8(3), 312-338 (2019)

Za'ba, N., Ismail, Z., y Abdullah, A. H., Preparing student teachers to teach mathematics with geogebra, https://doi.org/10.13189/ujer.2020.081904, Universal Journal of Educational Research, 8(5A), 29-33 (2020)

Zambak, V. S., y Tyminski, A. M., Examining mathematical technological knowledge of pre-service middle grades teachers with geometer's sketchpad in a geometry course, https://doi.org/10.1080/0020739X.2019.1650302, International Journal of Mathematical Education in Science and Technology, 51(2), 183-207 (2020)

Zambrano, C., Rojas, D., y Salcedo, P., Revisión sistemática a estudios de disponibilidad léxica en la base de datos Scielo y sus aportes a educación, http://dx.doi.org/10.4067/S0718-07642019000400189, Información Tecnológica., 30(4), 189-198 (2019) 
